\title{
Control of breathing in patients with limb girdle dystrophy: a controlled study
}

\author{
F Gigliotti, A Pizzi, R Duranti, M Gorini, I Iandelli, G Scano
}

\begin{abstract}
Background-In patients with limb girdle dystrophy the relative contribution of peripheral factors (respiratory muscle weakness, and lung and/or airway involvement) and central factors (blunted and/or inadequate chemoresponsiveness) in respiratory insufficiency has not yet been established. To resolve this, lung volumes, arterial blood gas tensions, respiratory muscle strength, breathing pattern and neural respiratory drive were investigated in a group of 15 patients with limb girdle dystrophy. An age-matched normal group was studied as a control.
\end{abstract}

Methods-Respiratory muscle strength was assessed as an arithmetic mean of maximal inspiratory (MIP) and expiratory (MEP) pressures. Breathing pattern was evaluated in terms of volume (ventilation VE, tidal volume VT) and time (respiratory frequency $R f$, inspiratory time $T I$, expiratory time TE) components of the respiratory cycle. Neural respiratory drive was assessed as the mean inspiratory flow (VT/TI), mouth occlusion pressure $\left(\mathbf{P}_{0.1}\right)$ and electromyographic activity (EMG) of the diaphragm (EMGd) and the intercostal parasternal (EMGp) muscles. In 10 of the 15 patients the responses to carbon dioxide $\left(\mathrm{PCO}_{2}\right)$ stimulation were also evaluated.

Results-Most patients exhibited a moderate decrease in vital capacity (VC) (range $37-87 \%$ of predicted), MIP (range 23-84\% of predicted), and/or MEP (range $13-41 \%$ of predicted). The arterial carbon dioxide tension $\left(\mathrm{PaCO}_{2}\right)$ was increased in three patients breathing room air, while $\mathrm{PaO}_{2}$ was normal in all. Compared with the control group $R f$ was higher, and VT, TI and TE were lower in the patients. EMGd and EMGp were higher whilst VT/ $T I$ and $P_{0.1}$ were normal in the patients. Respiratory muscle strength was inversely related to EMGd and EMGp. PaCO 2 was found to relate primarily to VC and duration of illness, but not to respiratory muscle strength. During hypercapnic rebreathing $\Delta V E / \Delta P C_{2}, \Delta V T / \Delta P O_{2}$, and $\Delta \mathbf{P}_{0.1} I \Delta \mathbf{P C O}_{2}$ were lower than normal, whilst $\Delta E M G d / \Delta P_{2} O_{2}$ and $\Delta E M G p / \Delta \mathrm{PCO}_{2}$ were normal in most patients. A direct relation between respiratory muscle strength and $\Delta V T / \Delta \mathrm{PCO}_{2}$ was found.

Conclusions-The respiratory muscles, especially expiratory ones, are weak in patients with limb girdle dystrophy. Reductions in respiratory muscle strength are associated with increased neural drive and decreased ventilatory output $(\Delta V T)$ $\left.\Delta \mathrm{PCO}_{2}\right)$. The decrease in VC, together with the duration of disease, influence $\mathrm{PaCO}_{2}$. VC is a more useful test than respiratory muscle strength for following the course of limb girdle dystrophy.

(Thorax 1995;50:962-968)

Keywords: neuromuscular diseases, respiratory drive, respiratory muscle strength, breathing pattern.

Patients with limb girdle dystrophy experience chronic cough, dyspnoea on exertion, and recurrent respiratory tract infection. ${ }^{1}$ Furthermore, chronic hypercapnia develops in the absence of precipitating factors such as pulmonary infection, oxygen therapy or sedation. ${ }^{23}$ Severe diaphragmatic weakness may be a factor contributing to the chronic hypercapnia, a problem which seems much more likely to develop in these patients than in those with Duchenne type dystrophy. ${ }^{4}$ However, no studies have been carried out to establish the relative contribution of peripheral (respiratory muscle weakness, and lung and/or airway involvement) and central (blunted and/or inadequate chemoresponsiveness) factors to respiratory insufficiency in patients with limb girdle dystrophy.

We have examined the relative importance of lung and chest wall mechanics and indices of central respiratory drive by measuring lung volumes, arterial blood gas tensions, overall respiratory muscle strength, breathing pattern, and hypercapnic ventilatory responses in a group of patients with limb girdle dystrophy and age matched controls.

\section{Methods}

SUBJECTS

Fifteen patients (seven men) of mean (SD) age $43.3(15.4)$ years (range 20-74) with limb girdle dystrophy and no respiratory complaints were studied; 12 were ambulatory and three were wheelchair bound. The following criteria updated from a previous classification ${ }^{5}$ were used to select patients: (1) inheritance pattern: autosomal recessive in six families and nine sporadic cases; (2) phenotype expression: all patients had a symmetrical limb girdle, proximal distribution without facial involvement or contractures. Distal limb muscles were involved only in the severely affected patients; (3) electromyographic findings were compatible with a progressive chronic myopathy; (4) histopathological findings in the muscles 
included dystrophic lesions without an inflammatory cell infiltrate or features of metabolic or congenital myopathies. In addition, in two sporadically affected men the absence of dystrophin abnormalities excluded Duchenne and Becker muscular dystrophies. No patients had a scoliosis or any abnormality on the chest radiograph or obvious abnormalities in diaphragm placement. ${ }^{4}$ Two patients were current smokers. The duration of their disease was $22 \cdot 2$ (9) years (range 8-35).

Twenty normal subjects matched for age (range 20-70 years, 10 men) were studied as a control group.

The study was approved by the local ethics committee and subjects gave their informed consent.

\section{FUNCTIONAL EVALUATION}

Routine spirometric tests and arterial blood gas tensions obtained with subjects in a seated position were measured as previously described. ${ }^{6}$ The normal values for lung volumes were those of the European Community for Coal and Steel. ${ }^{7}$ Maximal static inspiratory (MIP) and expiratory (MEP) pressures at residual volume (RV) and total lung capacity (TLC), respectively, measured against an obstructed mouthpiece with a small leak to minimise oral pressure artefacts, were recorded using a differential pressure transducer (Statham SC 1001). The subjects were comfortably seated, wearing a noseclip, and performed maximal inspiratory efforts maintaining maximal pressures for at least one second. The manoeuvres were repeated until three measurements with less than $5 \%$ variability were recorded, and the highest value obtained was taken for analysis. The predicted values for maximal respiratory pressures were those proposed by Black and Hyatt. ${ }^{8} \mathrm{~A}$ value of respiratory muscle strength was calculated as proposed by Braun et $a l^{9}$ [(MIP \% predicted value + MEP $\%$ predicted value) $/ 2]$. In the presence of respiratory muscle weakness the measured value of MIP and MEP may be affected by lung volume as well as by myopathy; we therefore corrected for these effects of lung volume on MIP and MEP. ${ }^{10}$

After baseline routine testing breathing room air the ventilatory pattern and mouth occlusion pressure were evaluated with subjects in a comfortable supine position. In the apparatus used the inspiratory line was separated from the expiratory line by a one-way valve (Hans-Rudolph) connected to a Fleisch type 3 pneumotachograph. The flow signal was integrated into volume. From the spirogram inspiratory time (TI), expiratory time ( $\mathrm{TE})$, total time of the respiratory cycle (TTOT), tidal volume (VT), mean inspiratory flow (VT/TI), and duty cycle (TI/Tтот) were derived. Respiratory frequency $(\mathrm{R} f=1 /$ TTOT $\times 60)$ and minute ventilation $(\mathrm{VE}=\mathrm{VT} \times \mathrm{R} f)$ were also calculated. Mouth pressure during VT manoeuvres was measured using a pressure transducer (Statham P23ID). Mouth occlusion pressure 0.1 second after the beginning of inspiration $\left(\mathrm{P}_{0.1}\right)^{11}$ was recorded as previously described. ${ }^{12-14}$ Expired $\mathrm{CO}_{2}\left(\mathrm{PCO}_{2}\right)$ was sampled continuously at the mouth and measured by an infrared $\mathrm{CO}_{2}$ meter (Normocap 200, Datex). The values for dead space and resistance of the system up to a flow of $4 \mathrm{l} / \mathrm{s}$ were $178 \mathrm{ml}$ and $0.92 \mathrm{~cm} \mathrm{H}_{2} \mathrm{O} / 1 / \mathrm{s}$, respectively.

The electromyographic activity (EMG) of the respiratory muscles was recorded as previously described. ${ }^{12-14}$ The EMG of the chest wall muscles was recorded from the second parasternal intercostal muscles (EMGp) and the diaphragm (EMGd) via large surface electrodes. The EMGd was recorded from the lower anterolateral rib cage. ${ }^{15}$ Muscle action potentials ("raw") were differentially amplified and filtered between 100 and $1000 \mathrm{~Hz}$ to remove as much ECG as possible without significantly filtering the EMG. The filtered EMG signal along with mouth pressure recording were displayed on a single beam storage oscilloscope (Tektronix 5115). EMG activity was full wave rectified and integrated over time (time constant $100 \mathrm{~ms}$ ) using a third order, low pass filter to provide a measurement of change in average electrical activity as a function of time, referred to as "moving time average". ${ }^{16}$ Inspiratory activity was quantified both as peak of activity (XP) and as rate of rise of activity $(\mathrm{XP} / \mathrm{TI})$. The former was directly measured in arbitrary units and the latter was obtained by dividing it by the inspiratory time.

Because of the variability of the impedance between diaphragm and electrodes, absolute values $(\mathrm{mV})$ are not comparable among different subjects. To overcome this problem and to obtain a reference value, EMG activity was measured while the subject, connected to the pneumotachograph, performed an inspiratory capacity (IC) manoeuvre breathing in up to the total lung capacity (TLC). ${ }^{12-14}$ This was repeated at least three times and in each subject the intensity of the recorded diaphragmatic EMG was closely reproducible (less than 5\% variability). The mean level of this EMG activity was taken as a reference; all successive measurements have been expressed as a percentage of this reference value obtained at TLC. As EMG activity of an inspiratory muscle may include cardiac muscle activity, we checked cardiac artefacts by manually gating the ECG, when necessary, so that it would not contribute to the EMG. EMG data in the text and tables are expressed in terms of rate of rise of activity.

The output of the $\mathrm{CO}_{2}$ meter, flow signal, integrated flow signal, mouth pressure, and the moving time average were recorded continuously on a multichannel chart recorder. After a 10 minute adaptation period, baseline evaluation began. Respiratory cycles, occlusions, and EMG were continuously recorded for 10 minutes and the cycles following occlusions discarded. Average values for each subject are presented. Subsequently, the subjects underwent a $\mathrm{CO}_{2}$ rebreathing test. ${ }^{17} \mathrm{~A}$ gas mixture $\left(7 \% \mathrm{CO}_{2}, 93 \% \mathrm{O}_{2}\right)$ was inhaled for 3-4 minutes and the sampled gas was returned to the rebreathing bag. Rebreathing started after 30-45 seconds which allowed the subject to equilibrate with the circuit, as shown by the plateau on the $\mathrm{CO}_{2}$ recording and minimal 
Table 1 Pulmonary function data in 15 patients with limb girdle dystrophy

\begin{tabular}{|c|c|c|c|c|c|c|c|c|c|c|c|}
\hline Subject & $\begin{array}{l}V C \\
\text { (\%pred) }\end{array}$ & $\begin{array}{l}F E V_{1} \\
\text { (\%pred) }\end{array}$ & $\begin{array}{l}F E V_{l} / V C \\
(\%)\end{array}$ & $\begin{array}{l}F R C \\
\text { (\%pred) }\end{array}$ & $\begin{array}{l}R V \\
\text { (\%pred) }\end{array}$ & $\begin{array}{l}\text { TLC } \\
\text { (\%pred) }\end{array}$ & $\begin{array}{l}\text { MIP } \\
\text { (\%pred) }\end{array}$ & $\begin{array}{l}\text { MEP } \\
\text { (\%pred) }\end{array}$ & $\begin{array}{l}R M S \\
(\%)\end{array}$ & $\begin{array}{l}\mathrm{PaO}_{2} \\
(\mathrm{kPa})\end{array}$ & $\begin{array}{c}\mathrm{PaCO}_{2} \\
(\mathrm{kPa})\end{array}$ \\
\hline $\begin{array}{r}1 \\
2 \\
3 \\
4 \\
5 \\
6 \\
7 \\
8 \\
9 \\
10 \\
11 \\
12 \\
13 \\
14 \\
15\end{array}$ & $\begin{array}{l}85 \\
68 \\
55 \\
68 \\
99 \\
71 \\
83 \\
37 \\
57 \\
90 \\
61 \\
37 \\
44 \\
57 \\
54\end{array}$ & $\begin{array}{l}83 \\
69 \\
57 \\
60 \\
82 \\
71 \\
83 \\
40 \\
62 \\
79 \\
64 \\
41 \\
45 \\
55 \\
52\end{array}$ & $\begin{array}{l}78 \\
88 \\
80 \\
70 \\
71 \\
81 \\
81 \\
81 \\
83 \\
77 \\
81 \\
94 \\
82 \\
78 \\
78\end{array}$ & $\begin{array}{l}86 \cdot 4 \\
88 \\
84 \\
79 \\
97 \\
92 \\
69 \\
59 \\
72 \\
81 \\
74 \\
68 \\
69 \\
95 \\
80 \cdot 5\end{array}$ & $\begin{array}{c}122 \cdot 7 \\
102 \\
98 \\
108 \\
100 \\
154 \\
152 \\
75 \\
159 \\
130 \\
100 \\
135 \\
97 \\
102 \\
99\end{array}$ & $\begin{array}{r}96 \\
80 \\
82 \\
88 \\
99 \\
100 \\
91 \\
62 \\
84 \\
100 \\
73 \\
65 \\
70 \\
86 \cdot 9 \\
82 \cdot 3\end{array}$ & $\begin{array}{l}52 \cdot 4 \\
64 \cdot 1 \\
92 \cdot 2 \\
49 \cdot 2 \\
68 \cdot 5 \\
79 \cdot 2 \\
89 \cdot 2 \\
36 \cdot 8 \\
84 \cdot 9 \\
89 \cdot 6 \\
73 \cdot 9 \\
27 \cdot 5 \\
46 \cdot 3 \\
29 \\
34 \cdot 5\end{array}$ & $\begin{array}{l}27 \cdot 5 \\
17 \cdot 8 \\
22 \\
12 \cdot 9 \\
32 \\
30 \\
24 \cdot 3 \\
14 \cdot 6 \\
34 \cdot 2 \\
27 \cdot 6 \\
42 \\
17 \cdot 9 \\
22 \cdot 1 \\
17 \cdot 2 \\
32 \cdot 9\end{array}$ & $\begin{array}{l}40 \\
41 \\
57 \cdot 1 \\
31 \cdot 1 \\
50 \cdot 5 \\
54 \cdot 6 \\
56 \cdot 8 \\
25 \cdot 7 \\
59 \cdot 6 \\
58 \cdot 6 \\
57 \cdot 9 \\
22 \cdot 7 \\
34 \cdot 2 \\
23 \cdot 1 \\
33 \cdot 7\end{array}$ & $\begin{array}{r}13.8 \\
11.7 \\
9.7 \\
13.5 \\
11.9 \\
13.3 \\
13.3 \\
10.2 \\
13.9 \\
12.7 \\
10.9 \\
10.1 \\
12.6 \\
10.5 \\
10.9\end{array}$ & $\begin{array}{l}4 \cdot 65 \\
5 \cdot 59 \\
5 \cdot 98 \\
4 \cdot 79 \\
5 \cdot 05 \\
4 \cdot 52 \\
4 \cdot 39 \\
6 \cdot 34 \\
5 \cdot 47 \\
5 \cdot 05 \\
5 \cdot 68 \\
6.09 \\
5 \cdot 72 \\
5 \cdot 88 \\
5 \cdot 32\end{array}$ \\
\hline $\begin{array}{l}\text { Mean } \\
\text { SD }\end{array}$ & $\begin{array}{l}64 \cdot 4 \\
18 \cdot 8\end{array}$ & $\begin{array}{l}62 \cdot 9 \\
14 \cdot 8\end{array}$ & $\begin{array}{r}80 \cdot 2 \\
5 \cdot 9\end{array}$ & $\begin{array}{l}79 \cdot 6 \\
11.0\end{array}$ & $\begin{array}{l}115 \\
25 \cdot 1\end{array}$ & $\begin{array}{l}83 \cdot 9 \\
12 \cdot 4\end{array}$ & $\begin{array}{l}61 \cdot 1 \\
23 \cdot 3\end{array}$ & $\begin{array}{r}25 \cdot 0 \\
8 \cdot 3\end{array}$ & $\begin{array}{l}43 \cdot 1 \\
14 \cdot 0\end{array}$ & $\begin{array}{r}11.9 \\
1.5\end{array}$ & $\begin{array}{l}5 \cdot 37 \\
0.60\end{array}$ \\
\hline
\end{tabular}

$\mathrm{VC}=$ vital capacity; $\mathrm{FEV}_{1}=$ forced expiratory volume in one second; $\mathrm{FRC}=$ functional residual capacity; $\mathrm{RV}=$ residual volume; $\mathrm{TLC}=$ total lung capacity; $\mathrm{MIP}=$ maximal inspiratory pressure; $\mathrm{MEP}=$ maximal expiratory pressure: $\mathrm{RMS}=$ respiratory muscle strength calculated as arithmetical mean of $\mathrm{MIP}$ (\% predicted) and MEP (\% predicted); $\mathrm{PaO}_{2}=$ arterial oxygen tension; $\mathrm{PaCO}_{2}=$ arterial carbon dioxide tension.

$\mathrm{PCO}_{2}$ inspiratory/expiratory swings. The resistance of the circuit used during unoccluded breathing was such that mouth pressure during unoccluded breathing was always within $<0.2 \mathrm{kPa}$ of atmospheric pressure. During $\mathrm{CO}_{2}$ inhalation, when the open loop condition was achieved, occlusions were randomly performed every 10-20 seconds. Both in normal subjects and in patients peak average at TLC remained fairly constant throughout the $\mathrm{CO}_{2}$ rebreathing run $(5-10 \%)$. In each subject the test was repeated on the same day with an interval of at least 60 minutes between each test. For each rebreathing run changes in $\mathrm{VE}$, time, and volume components of breathing pattern, $\mathbf{P}_{0.1}$ and EMG were plotted against increasing $\mathrm{PCO}_{2}$ and subjected to least square linear regression analysis.

Rebreathing data were available for 10 out of the 15 patients (nos 1-5, 11-15), since two of the remaining five did not give their consent to be tested and three patients (nos 6, 8, and 9) sensed such discomfort during inhalation of the $\mathrm{CO}_{2}$ mixture that it caused early interruption of the test.

DATA ANALYSIS

The results were compared by the Mann-Whitney $\mathrm{U}$ test for unpaired samples. The relation between respiratory muscle strength and VC, $\mathrm{PaCO}_{2}, \mathrm{EMG}$, and $\mathrm{VE}$ and VT response slope to increasing $\mathrm{CO}_{2}$ were assessed by linear regression analysis. The relation between different variables was analysed by multiple regression analysis, a value of $\mathrm{p}<0.05$ being considered to be significant. All statistical analyses were carried out using the Statgraphics 6.0 package (Manugistics Inc, Rockville, MD, USA).

\section{Results}

DATA UNDER CONTROL CONDITIONS Spirometry and arterial blood gas tensions

Lung function, muscle strength, and arterial blood gas tensions for the patients are summarised in table 1 . The patients had a decreased vital capacity (VC, $64 \%$ of predicted), forced expiratory volume in one second $\left(\mathrm{FEV}_{1}, 62.9 \%\right.$ of predicted), and functional residual capacity (FRC, $79.6 \%$ of predicted) with a small increase in residual volume (RV, $115 \%$ of predicted). $\mathrm{FEV}_{1} / \mathrm{VC} \%$ was normal in all patients. A moderate (55\% to $40 \%$ predicted value) to marked ( $<40 \%$ predicted value) decrease in MIP was observed in seven patients (nos 1, 4, $8,12-15)$, and a minimal change ( $75 \%$ to $55 \%$ predicted value) was observed in four (nos 2 , $5,6,11)$; the decrease in MEP was more marked in all subjects. Arterial oxygen tension $\left(\mathrm{PaO}_{2}\right)$ was normal in all but three patients (nos $3,8,14$ ) while arterial carbon dioxide tension $\left(\mathrm{PaCO}_{2}\right)$ was increased in patients 3, 8, and 12 .

\section{BREATHING PATTERN}

Table 2 shows the breathing pattern in the patient group compared with the normal control group. In the patients VT $(p<0.005)$, TI, $\mathrm{TE}$, and Tтот $(\mathrm{p}<0.05)$ were lower, and $\mathrm{R} f$ higher $(p<0.05)$. In particular, in seven patients (nos 6-8, 11-13, and 15) VT was less than or equal to the mean $-2 S D$ of the value calculated for the control group. No significant difference between the two groups was observed for the $\mathrm{VT} / \mathrm{TI}$ and $\mathrm{TI} / \mathrm{TTOT}$ ratios.

\section{Respiratory drive}

Mouth occlusion pressure $\left(P_{0.1}\right)$ and EMG activity $(\mathrm{XP} / \mathrm{TI})$ during room air breathing are also reported in table 2 . Compared with the control group, the $P_{0.1}$ was similar but the EMGd and EMGp were higher in the patients ( $p<0.05$ for both).

Significant relationships were observed between respiratory muscle strength and EMGd $(r=-0.7, \mathrm{p}<0.005)$ (fig 1A), EMGp $(r=$ $-0.66, \mathrm{p}<0.01)$, and VC $(r=0.55, \mathrm{p}<0.03)$ (fig 1B); VC also related to EMGd $(r=-0.69$, $\mathrm{p}<0.01)$. In addition, stepwise regression analysis showed that VC and disease duration together predicted about $82 \%$ of variability in $\mathrm{PaCO}_{2}$ (table 3).

\section{DATA FROM $\mathrm{CO}_{2}$ REBREATHING}

The ventilatory, $P_{0.1}$ and EMG responses to increasing $\mathrm{PCO}_{2}$ for the two groups are sum- 
Table 2 Breathing pattern, mouth occlusion pressure, and electromyographic activity of the diaphragm and intercostal muscles in 15 patients with limb girdle dystrophy

\begin{tabular}{|c|c|c|c|c|c|c|c|c|c|c|c|}
\hline Patients & $\begin{array}{l}V E \\
(I / \text { min })\end{array}$ & $\begin{array}{l}R f \\
\text { (cycles/min) }\end{array}$ & $\begin{array}{l}V T \\
\text { (l) } \\
\end{array}$ & $\begin{array}{l}T I \\
(s)\end{array}$ & $\begin{array}{l}T E \\
(s)\end{array}$ & $\begin{array}{l}\text { TTOT } \\
\text { (s) }\end{array}$ & $\begin{array}{l}V T / T I \\
(l / s)\end{array}$ & TI/TTOT & $\begin{array}{l}P_{0 \cdot 1} \\
\left(\mathrm{~cm} \mathrm{H} \mathrm{H}_{2} \mathrm{O}\right)\end{array}$ & $\begin{array}{l}E M G d \\
(\% T L C / s)\end{array}$ & $\begin{array}{l}E M G p \\
(\% T L C / s)\end{array}$ \\
\hline $\begin{array}{r}1 \\
2 \\
3 \\
4 \\
5 \\
6 \\
7 \\
8 \\
9 \\
10 \\
11 \\
12 \\
13 \\
14 \\
15\end{array}$ & $\begin{array}{r}9 \cdot 17 \\
12 \cdot 7 \\
9 \cdot 7 \\
7 \cdot 0 \\
8 \cdot 3 \\
9 \cdot 2 \\
7 \cdot 5 \\
7 \cdot 8 \\
7 \cdot 5 \\
13 \cdot 6 \\
8 \cdot 8 \\
8 \cdot 1 \\
9 \cdot 9 \\
11 \cdot 8 \\
7 \cdot 8\end{array}$ & $\begin{array}{l}14 \\
22 \\
11 \\
11 \\
13 \\
18 \\
18 \\
26 \\
14 \\
20 \\
19 \\
20 \\
23 \\
22 \\
16\end{array}$ & $\begin{array}{l}0.67 \\
0.57 \\
0.85 \\
0.63 \\
0.63 \\
0.51 \\
0.44 \\
0.30 \\
0.60 \\
0.70 \\
0.46 \\
0.47 \\
0.43 \\
0.55 \\
0.50\end{array}$ & $\begin{array}{l}1.82 \\
1.17 \\
2.13 \\
2.1 \\
1.65 \\
1.24 \\
1.62 \\
0.74 \\
1.5 \\
1.45 \\
1.55 \\
1.08 \\
1.14 \\
1.2 \\
1.47\end{array}$ & $\begin{array}{l}2.6 \\
1.6 \\
3.3 \\
3.4 \\
2.9 \\
2.1 \\
2.2 \\
1.5 \\
2.5 \\
1.6 \\
1.6 \\
2.0 \\
1.5 \\
1.58 \\
2.3\end{array}$ & $\begin{array}{l}4 \cdot 4 \\
2 \cdot 76 \\
5 \cdot 4 \\
5 \cdot 5 \\
4 \cdot 5 \\
3 \cdot 5 \\
3 \cdot 4 \\
2 \cdot 3 \\
4 \cdot 0 \\
3 \cdot 1 \\
3 \cdot 2 \\
3 \cdot 1 \\
2 \cdot 65 \\
2 \cdot 78 \\
3 \cdot 77\end{array}$ & $\begin{array}{l}0.37 \\
0.49 \\
0.41 \\
0.29 \\
0.38 \\
0.40 \\
0.27 \\
0.4 \\
0.4 \\
0.48 \\
0.3 \\
0.39 \\
0.38 \\
0.45 \\
0.34\end{array}$ & $\begin{array}{l}0.41 \\
0.43 \\
0.39 \\
0.40 \\
0.36 \\
0.35 \\
0.48 \\
0.32 \\
0.38 \\
0.46 \\
0.48 \\
0.35 \\
0.43 \\
0.43 \\
0.4\end{array}$ & $\begin{array}{l}0.8 \\
1.1 \\
1.5 \\
1.0 \\
1.3 \\
1.2 \\
1.3 \\
1.1 \\
1.4 \\
1.2 \\
1.2 \\
1.1 \\
1.2 \\
1.0 \\
1.4\end{array}$ & $\begin{array}{c}2 \\
14 \cdot 3 \\
5 \cdot 4 \\
2 \cdot 1 \\
5 \\
2 \cdot 5 \\
4 \cdot 8 \\
59 \\
5 \cdot 1 \\
6 \cdot 2 \\
9 \\
31 \cdot 9 \\
8 \cdot 6 \\
40 \\
20\end{array}$ & $\begin{array}{c}11 \cdot 4 \\
15 \cdot 6 \\
6 \cdot 7 \\
10 \\
10 \cdot 6 \\
5 \\
11 \cdot 1 \\
81 \\
8 \cdot 5 \\
10 \cdot 2 \\
8 \cdot 8 \\
18 \cdot 8 \\
27 \cdot 9 \\
49 \\
25\end{array}$ \\
\hline $\begin{array}{l}\text { Mean } \\
\text { SD }\end{array}$ & $\begin{array}{l}9 \cdot 10 \\
2 \cdot 10\end{array}$ & $\begin{array}{c}17 \cdot 8 \# \\
4 \cdot 5\end{array}$ & $\begin{array}{l}0.55 \dagger \\
0.14\end{array}$ & $\begin{array}{l}1.46^{*} \\
0.4\end{array}$ & $\begin{array}{l}2 \cdot 20^{*} \\
0.65\end{array}$ & $\begin{array}{l}3.62^{*} \\
0.9\end{array}$ & $\begin{array}{l}0.38 \\
0.06\end{array}$ & $\begin{array}{l}0.40 \\
0.042\end{array}$ & $\begin{array}{l}1 \cdot 20 \\
0 \cdot 18\end{array}$ & $\begin{array}{l}14 \cdot 4^{*} \\
16 \cdot 7\end{array}$ & $\begin{array}{l}19 \cdot 9^{*} \\
20.0\end{array}$ \\
\hline \multicolumn{12}{|l|}{$\begin{array}{l}\text { Control } \\
\text { values }\end{array}$} \\
\hline
\end{tabular}

$\mathrm{VE}=$ minute ventilation; $R f=$ respiratory frequency; $\mathrm{VT}=$ tidal volume; $\mathrm{TI}=$ inspiratory time; $\mathrm{TE}=$ expiratory time; $\mathrm{TTOT}=$ total time of the respiratory cycle; $\mathrm{P}_{0.1}=$ mouth occlusion pressure; EMGd = electromyographic activity of the diaphragm; EMGp = electromyographic activity of the parasternal muscles. EMG is expressed as ratio of rise of inspiratory activity obtained by dividing peak activity by the relevant inspiratory time (XP/TI).

* $p<0.05 ; \# p<0.025 ;+p<0.005$.

Table 3 Stepwise linear multiple regression analysis for $\mathrm{PaCO}_{2}$

\begin{tabular}{|c|c|c|c|}
\hline Variable & Coefficient & $\begin{array}{l}\text { Model } r^{2} \\
(\%)\end{array}$ & $p$ \\
\hline \multirow{2}{*}{$\begin{array}{l}\text { Constant } \\
\text { VC } \\
\text { Duration of } \\
\text { disease }\end{array}$} & $\begin{array}{c}46 \cdot 8 \\
-0 \cdot 18\end{array}$ & 0.61 & 0.0001 \\
\hline & 0.22 & 0.82 & 0.003 \\
\hline
\end{tabular}

$\mathrm{VC}=$ vital capacity.

marised in table 4. As a mean, $\Delta(\mathrm{VT} / \mathrm{TI}) / \Delta \mathrm{PcO}_{2}$ was similar in the patients and the controls while $\Delta \mathrm{VE} / \Delta \mathrm{PCO}_{2}, \Delta \mathrm{VT} / \Delta \mathrm{PCO}_{2} \quad(\mathrm{p}<0.05$ for both) and $\Delta \mathrm{P}_{0.1} / \Delta \mathrm{PcO}_{2}(\mathrm{p}<0.01)$ were significantly lower in the patients; $\triangle \mathrm{EMGd} / \Delta \mathrm{PCO}_{2}$ and $\Delta \mathrm{EMGp} / \Delta \mathrm{PCO}_{2}$ did not differ between the two groups. Also, unlike the normal group, a significant relation between increasing $\mathrm{PCO}_{2}$ and $R f$ (see table 4) was found in most patients. Table 5 shows VE, Rf, VT, VT/TI, $P_{0.1}$, and

A

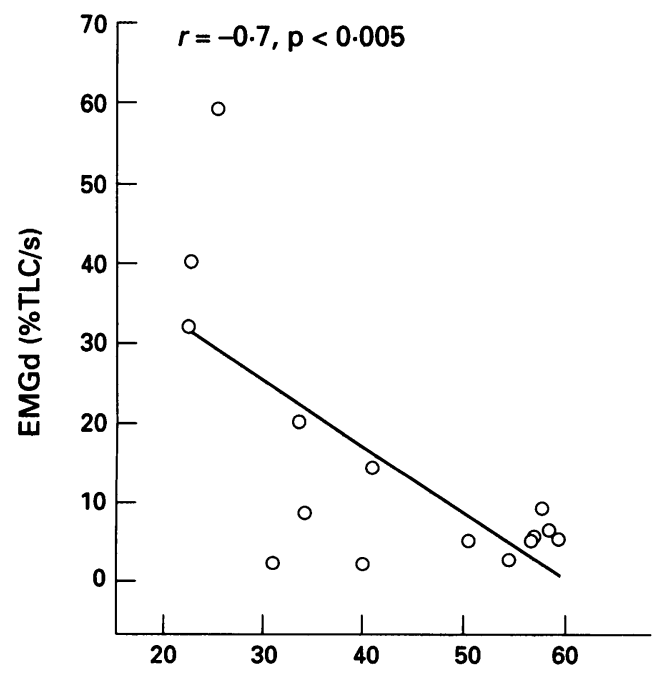

EMG values during $\mathrm{CO}_{2}$ rebreathing at $8 \mathrm{kPa}$ of $\mathrm{PCO}_{2}$. Patients exhibited significantly lower VT and higher $R f$ and EMGd ( $\mathrm{p}<0.05$ for all comparisons), reflecting the behaviour while they breathed room air. The lower $P_{0.1}$ value $(p<0.05)$ reflected the flat $\Delta \mathrm{P}_{0.1} / \Delta \mathrm{PCO}_{2}$.

Respiratory muscle strength related to $\Delta \mathrm{VT} /$ $\Delta \mathrm{PcO}_{2}(r=0.68, \mathrm{p}<0.03)$ (fig 2$)$ and tended to be related to $\Delta \mathrm{VE} / \Delta \mathrm{PCO}_{2}(r=0.62, \mathrm{p}=\mathrm{NS})$. However, neither the relationship of $\mathrm{VC}$ with $\Delta \mathrm{VT} / \Delta \mathrm{PCO}_{2}$ nor the relation of respiratory muscle strength to $\Delta(\mathrm{VT} / \mathrm{TI}) / \Delta \mathrm{PcO}_{2}$ or $\Delta \mathrm{P}_{0.1} /$ $\Delta \mathrm{PCO}_{2}$ was significant.

\section{Discussion}

PULMONARY VOLUMES AND RESPIRATORY MUSCLE STRENGTH

Few studies have addressed the issue of respiratory abnormalities in patients with limb girdle dystrophy. In patients with neuro-

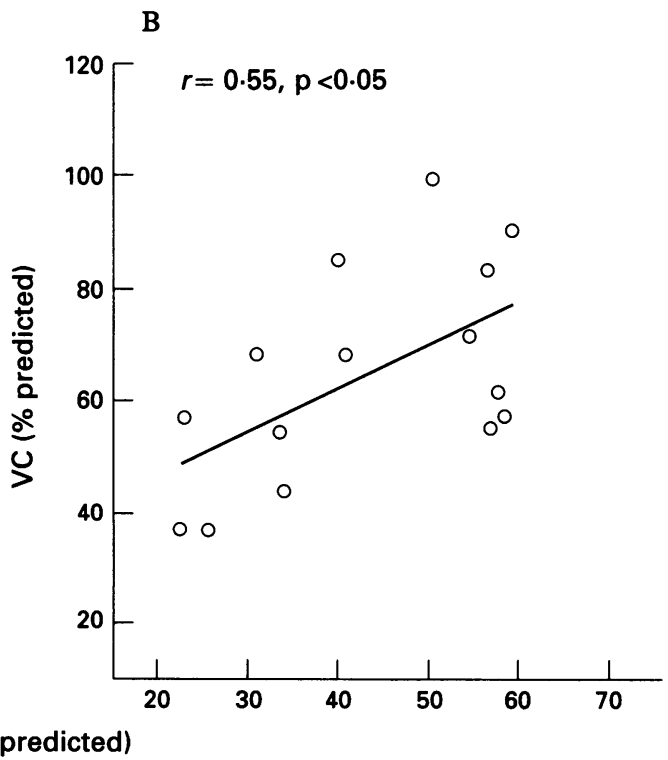

Figure 1 Relation of respiratory muscle strength (RMS) to (A) electromyographic activity of the diaphragm (EMGd) and (B) vital capacity (VC). 
Table $4 V E, R f, V T, V T / T I, P_{0 \cdot 1}$, and EMG response slopes during $C_{2}$ rebreathing in 10 patients with limb girdle dystrophy compared with the control group

\begin{tabular}{|c|c|c|c|c|c|c|c|}
\hline Patients & $\begin{array}{l}\triangle V_{E} / \triangle P C O_{2} \\
((a / m i n) / k P a)\end{array}$ & $\begin{array}{l}\triangle V T / \Delta P C O_{2} \\
(a / k P a)\end{array}$ & $\begin{array}{l}\triangle R f / \triangle P C O_{2} \\
((b r e a t h s / \min ) / \\
k P a)\end{array}$ & $\begin{array}{l}\Delta(V T / T) / \Delta P C O_{2} \\
((l / s) / k P a))\end{array}$ & $\begin{array}{l}\Delta P_{0 \cdot 1} / \Delta P \mathrm{PO}_{2} \\
\left(\mathrm{~cm} \mathrm{H} \mathrm{H}_{2} \mathrm{O} / \mathrm{kPa}\right)\end{array}$ & $\begin{array}{l}\triangle E M G d / \triangle P C O_{2} \\
((\% T L C / s) / k P a)\end{array}$ & $\begin{array}{l}\triangle E M G p / \Delta P C O_{2} \\
((\% T L C / s) / k P a)\end{array}$ \\
\hline $\begin{array}{r}1 \\
2 \\
3 \\
4 \\
5 \\
11 \\
12 \\
13 \\
14 \\
15\end{array}$ & $\begin{array}{c}10 \cdot 3 \\
3 \cdot 61 \\
19 \cdot 2 \\
4 \cdot 89 \\
9 \cdot 10 \\
9 \cdot 77 \\
4 \cdot 13 \\
3 \cdot 38 \\
9 \cdot 02 \\
7 \cdot 52\end{array}$ & $\begin{array}{l}0.52 \\
0.13 \\
0.66 \\
0.41 \\
0.36 \\
0.45 \\
0.13 \\
0.15 \\
0.19 \\
0.29\end{array}$ & $\begin{array}{l}0.31 \\
3 \cdot 01 \\
7 \cdot 51 \\
-1 \cdot 35 \\
2 \cdot 76 \\
\frac{-}{3 \cdot 16} \\
2 \cdot 78\end{array}$ & $\begin{array}{l}0.34 \\
0 \cdot 16 \\
0.68 \\
0.30 \\
0 \cdot 23 \\
0.30 \\
0 \cdot 19 \\
0.19 \\
0.47 \\
0.49\end{array}$ & $\begin{array}{l}1.43 \\
1.13 \\
2.10 \\
0.45 \\
1.05 \\
1.50 \\
0.98 \\
0.53 \\
1.43 \\
1.05\end{array}$ & $\begin{array}{c}6 \cdot 77 \\
9 \cdot 10 \\
14 \cdot 3 \\
7 \cdot 52 \\
5 \cdot 56 \\
16 \cdot 4 \\
13 \cdot 3 \\
1 \cdot 50 \\
6 \cdot 84 \\
8 \cdot 27\end{array}$ & $\begin{array}{c}5.04 \\
41.9 \\
24.8 \\
- \\
5.04 \\
35.3 \\
14.7 \\
6.84 \\
13.5 \\
21.8\end{array}$ \\
\hline $\begin{array}{l}\text { Mean } \\
\text { SD } \\
\text { Control values }\end{array}$ & $\begin{array}{l}8 \cdot 10^{*} \\
4 \cdot 74\end{array}$ & $\begin{array}{l}0.33^{*} \\
0 \cdot 18\end{array}$ & $\begin{array}{l}2 \cdot 98 \\
2 \cdot 25\end{array}$ & $\begin{array}{l}0 \cdot 33 \\
0 \cdot 16\end{array}$ & $\begin{array}{l}1 \cdot 16 \ddagger \\
0 \cdot 48\end{array}$ & $\begin{array}{l}8.95 \\
4 \cdot 49\end{array}$ & $\begin{array}{l}18 \cdot 8 \\
13 \cdot 3\end{array}$ \\
\hline $\begin{array}{l}\text { Mean } \\
\text { SD }\end{array}$ & $\begin{array}{c}13 \cdot 5^{*} \\
3 \cdot 0\end{array}$ & $\begin{array}{l}0 \cdot 64^{*} \\
0 \cdot 10\end{array}$ & - & $\begin{array}{l}0.40 \\
0.08\end{array}$ & $\begin{array}{l}2.63 \ddagger \\
0.60\end{array}$ & $\begin{array}{r}10.5 \\
3.0\end{array}$ & $\begin{array}{c}10 \cdot 6 \\
3.01\end{array}$ \\
\hline
\end{tabular}

Abbreviations as in table 3. EMG is expressed as ratio of rise of inspiratory activity obtained by dividing peak activity by the relevant inspiratory time (XP/TI). $* \mathrm{p}<0 \cdot 05 ; \neq \mathrm{p}<0 \cdot 01$.

Table $5 V E, R f, V T, V T / T I, P_{0 \cdot 1}, E M G d$, and EMGp values during $\mathrm{CO}_{2}$ rebreathing at a $\mathrm{PCO}_{2}$ of $8 \mathrm{kPa}$

\begin{tabular}{|c|c|c|c|c|c|c|c|}
\hline & $\begin{array}{l}V E \\
(\text { limin) }\end{array}$ & $\begin{array}{l}R f \\
\text { (breaths/min) }\end{array}$ & $\begin{array}{l}V T \\
\text { (l) }\end{array}$ & $\begin{array}{l}V T / T I \\
(l / s)\end{array}$ & $\begin{array}{l}P_{0 \cdot 1} \\
\left(\mathrm{~cm} \mathrm{H} \mathrm{H}_{2} \mathrm{O}\right)\end{array}$ & $\begin{array}{l}\text { EMGd } \\
(\% T L C / s)\end{array}$ & $\begin{array}{l}E M G p \\
(\% T L C / s)\end{array}$ \\
\hline $\begin{array}{l}\text { Limb girdle } \\
\text { dystrophy } \\
\text { Control }\end{array}$ & $\begin{array}{c}21 \cdot 5 \\
(8 \cdot 8) \\
24 \cdot 0 \\
(6 \cdot 0)\end{array}$ & $\begin{array}{l}21 \cdot 8^{*} \\
(8 \cdot 3) \\
15 \cdot 0^{*} \\
(4 \cdot 3)\end{array}$ & $\begin{array}{c}0.98^{*} \\
(0.5) \\
1.6^{*} \\
(0.41)\end{array}$ & $\begin{array}{c}0.6 \\
(0.19) \\
0.75 \\
(0.21)\end{array}$ & $\begin{array}{c}2 \cdot 9^{*} \\
(0 \cdot 68) \\
5 \cdot 40^{*} \\
(2 \cdot 6)\end{array}$ & $\begin{array}{c}19 \cdot 7^{*} \\
(11 \cdot 2) \\
9 \cdot 6^{*} \\
(4 \cdot 8)\end{array}$ & $\begin{array}{c}29 \cdot 5 \\
(21 \cdot 0) \\
11 \\
(5)\end{array}$ \\
\hline
\end{tabular}

Values are mean $\pm 1 \mathrm{SD}$. Abbreviations as in table 3. EMG is expressed as rate of rise of inspiratory activity obtained by dividing peak activity by the relevant inspiratory time (XP/TI) $* \mathrm{p}<0.05$.

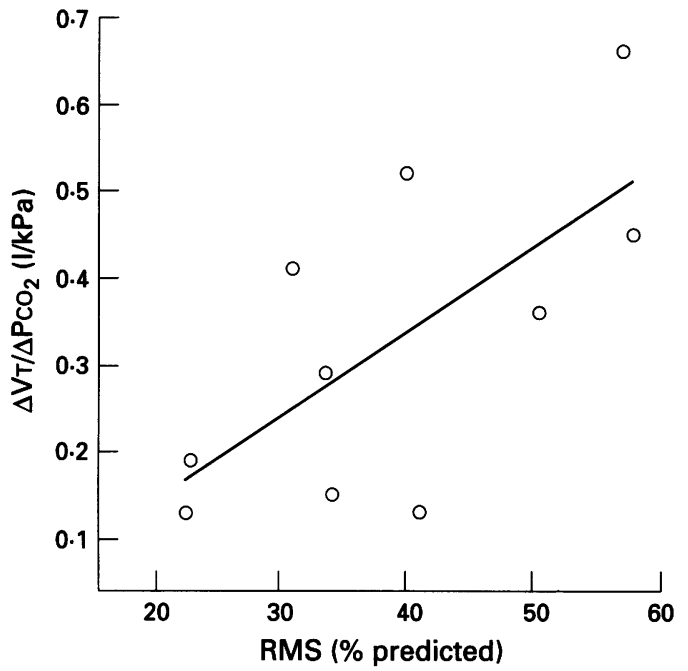

Figure 2 Relation of respiratory muscle strength (RMS) to $\triangle V T / \triangle P C O_{2}$. VT = tidal volume; $\mathrm{PCO}_{2}=$ carbon dioxide tension.

muscular diseases, including 15 patients with limb girdle dystrophy, without respiratory symptoms, the restrictive defect was attributed to a decrease in respiratory muscle strength. ${ }^{18}$ The extent of expiratory muscle weakness and the relative contribution of expiratory muscles in that study was similar to our present data. In our study respiratory muscle strength predicted $30.2 \%$ of the variance in VC so that the decrease in VC was out of proportion to the decrease in respiratory muscle strength as predicted from theoretical considerations. ${ }^{19}$ This confirms that factors other than respiratory muscle weakness itself are implicated in the loss of lung volume. ${ }^{2021}$

In our patients the level of $\mathrm{PaCO}_{2}$ at rest was not related to respiratory muscle strength, and is consistent with the observations by Braun et $a l$ in patients with metabolic myopathies where respiratory muscle strength was $>50 \%$ of predicted. ${ }^{9}$ On the other hand, stepwise regression analysis showed that VC and duration of illness accounted for $82 \%$ of the variability in $\mathrm{PaCO}_{2}$. In this sense we agree that VC is a more useful test than respiratory muscle strength to follow the course of the neuromuscular disease ${ }^{20}$ No data are available on the histological features of the diaphragm and the other respiratory muscles in limb girdle dystrophy. ${ }^{22}$ Thus, the mechanism of respiratory muscle weakness remains to be clarified.

\section{BREATHING PATTERN}

Compared with the normal control group, patients showed an increase in $R f$ and a decrease in VT while breathing room air, a common feature in patients with neuromuscular disease. ${ }^{143-27}$ The decrease in VT was associated with a significant reduction in inspiratory time (TI). During exogenous $\mathrm{CO}_{2}$ stimulation $\Delta \mathrm{VE} / \Delta \mathrm{PCO}_{2}$ was lower in the patients as they responded to increasing $\mathrm{PCO}_{2}$ with a similar increase in VT and a compensatory increase in $\mathrm{Rf}\left(\Delta \mathrm{Rf} / \Delta \mathrm{PcO}_{2}\right.$, see table 4). At a $\mathrm{PCO}_{2}$ of $8 \mathrm{kPa}$ the breathing pattern reflected the general behaviour shown during room air breathing. Reduction in lung compliance, ${ }^{26}$ a stiffer rib cage, ${ }^{20}$ and respiratory muscle weakness ${ }^{23}$ are thought to be important factors which contribute to rapid and shallow breathing. Respiratory muscle weakness might contribute to the perception of respiratory effort in patients with neuromuscular disease and so to the reduction in VT. ${ }^{28}$ In contrast, the significant relationship between respiratory muscle strength and $\Delta \mathrm{VT} / \Delta \mathrm{PCO}_{2}$ (fig 2) suggests 
that respiratory muscle weakness contributes to the abnormal VT response during hypercapnic stimulation in patients with limb girdle dystrophy.

NEURAL RESPIRATORY DRIVE

In addition to traditional measurements for assessing neural respiratory drive $(\mathrm{VT} / \mathrm{TI}$ and $\left.P_{0.1}\right), E M G$ of the respiratory muscles was also employed. We have criticised the use of either surface or oesophageal EMG recordings for assessing neural drive in humans. ${ }^{12-14}$ However, our data support the contention that the rate of rise of the "moving time average" is reliable for assessing neural inspiratory drive to the respiratory muscles both in normal cases and in disease. ${ }^{12-14162930}$

\section{Room air breathing}

Compared with the normal group, our patients showed a greater fractional electromyographic activity of both the diaphragm (EMGd) and the intercostal parasternal (EMGp) muscles. This activity indicates the extent of its activation per breath relative to its total available activity. We have previously noted higher fractional EMG activity in patients with other respiratory diseases in which the inspiratory drive is expected to increase. ${ }^{12-14}$ In patients with respiratory muscle weakness, a measure of muscle output $\left(\mathrm{P}_{0 \cdot 1}\right)^{31}$ or its translation into inspiratory flow $(\mathrm{VT} / \mathrm{TI})^{24}$ may underestimate the neural respiratory drive. The observation that spontaneously breathing patients with severe respiratory muscle weakness had normal $\mathrm{VT} / \mathrm{TI}$ and $\mathrm{P}_{0.1}$ and high levels of EMG activity is therefore likely to reflect, in line with previous studies, an increased neural activation of the inspiratory muscles. ${ }^{923-2533}$ In this connection it has been suggested that, in patients with neuromuscular disorders, neural drive could be increased in response to respiratory muscle weakness or loading. ${ }^{3233334}$ This is how we interpret the inverse relationships between respiratory muscle strength and both EMGd (fig 1) and EMGp.

\section{Response to $\mathrm{CO}_{2}$ stimulation}

The low $\Delta \mathrm{P}_{0.1} / \Delta \mathrm{PCO}_{2}$ is in line with previous studies where respiratory drive was suspected to be either reduced ${ }^{3536}$ or inadequate, ${ }^{31}$ even when the decrease in respiratory muscle strength was minimal, ${ }^{35}$ but it is at variance with others ${ }^{2324}$ where a normal $P_{0 \cdot 1}$ and low VT and $\mathrm{VT} / \mathrm{TI}$ responses to carbon dioxide were thought to indicate a normal drive. In the present study the low $\Delta \mathrm{P}_{0.1} / \Delta \mathrm{PCO}_{2}$ was in sharp contrast with the normality of $\Delta(\mathrm{VT} / \mathrm{TI}) / \Delta \mathrm{PCO}_{2}$ and both $\Delta \mathrm{EMGd} / \Delta \mathrm{PCO}_{2}$ and $\Delta \mathrm{EMGp} / \Delta \mathrm{PCO}_{2}$. $\mathrm{VT} / \mathrm{TI}$ is not sensitive to changes in elastic lung properties $^{37}$ or imposition of elastic loading, ${ }^{30}$ both conditions resulting in an increased respiratory drive which maintains VT/TI constant. Based on those observations, in patients in whom loss of VC disproportionate to the decrease in respiratory muscle strength may suggest abnormalities in elastic properties of the lung, ${ }^{20}$ a relatively normal VT/TI would suggest an increased neural respiratory drive. It is also possible that the decrease in $\Delta(\mathrm{VT} / \mathrm{TI}) / \Delta \mathrm{PCO}_{2}$ reflects a decrease in respiratory muscle strength rather than a low neural respiratory drive. Although this could not be totally excluded in the present investigation, no significant relation was found between $\Delta(\mathrm{VT} / \mathrm{TI}) /$ $\Delta \mathrm{PCO}_{2}$ and respiratory muscle strength. In fact, in the patients with a low VT/TI response, the respiratory muscle strength was similar to that observed in those with a normal VT/TI response $(34 \cdot 11(10 \cdot 7) \%$ versus $36 \cdot 9 \%(12 \cdot 15) \%)$.

The explanation of a markedly low $\Delta \mathrm{P}_{0 \cdot 1} /$ $\Delta \mathrm{PCO}_{2}$ is not clear. It is unlikely that, in the circumstances of the present study, the decrease in respiratory muscle strength could be the reason for the low $\Delta \mathrm{P}_{0.1} / \Delta \mathrm{PcO}_{2}$. In fact, the $\mathrm{P}_{0.1}$ response to carbon dioxide did not relate to muscle weakness.

A clue might be the measurement of $P_{0.1}$, the phase lag between pressure and flow and the shape of the driving pressure being some of the factors which should be considered in evaluating $P_{0 \cdot 1}$ as an index of respiratory drive. Phase lag between pressure and flow may occur as a consequence of resistance just at the end of expiration. This may be due to abnormal function of the upper airway muscles which is common in neuromuscular disease. ${ }^{38}$ When ventilation increases, as during chemically stimulated breathing, the accessory inspiratory muscles and the expiratory muscles are recruited and changes in the coordinated action of the respiratory muscles can lead to upper airways obstruction. ${ }^{39}$ As a consequence of phase lag, $P_{0.1}$ may be either higher or lower than the rate of increase in pressure at the beginning of inspiration, depending on whether the pressure wave in early inspiration is concave or convex. ${ }^{40}$ Thus, a change in shape of the pressure wave is not unexpected in patients with neuromuscular disease. ${ }^{40}$

In summary, in patients with limb girdle dystrophy a decrease in VC, together with the duration of disease, seems to influence the $\mathrm{PaCO}_{2}$. Peripheral factors (respiratory muscle weakness) and, in a few circumstances, blunted or inadequate chemoresponsiveness may account for the reduced $\mathrm{VE}$ and $\mathrm{VT}$ response during high levels of chemical stimulation.

1 Kilburn KH, Eagen JT, Sieker HO, Heyman A. Cardiopulmonary insufficiency in myotonic and progressive muscular dystrophy. $N$ Engl f Med 1959;261:1089-96.

2 Neustadt JE, Levy RC, Spiegel IJ. Carbon dioxide narcosis in association with muscular dystrophy. $\mathscr{f} A M A$ 1964;187: 616-7.

3 Newsom-Davies J, Goldman M, Loh L, Casson M. Diaphragm function and alveolar hypoventilation. $Q \mathcal{F} \mathrm{Med}$ phragm function

4 Newsom-Davies J. The respiratory system in muscular dystrophy. Br Med Bull 1980;36:135-8.

5 Walton JN, Natrass FJ. On the classification, natural history and treatment of the myopathies. Brain 1954;77:12-231.

6 Scano G, Garcia Herreros P, Stendardi D, De Coster A Sergysels R. Cardiopulmonary adaptation to exercise in coal miners. Arch Environ Health 1980;35:360-6.

7 European Community for Coal and Steel. Standardization of lung function tests. Eur Respir $\mathcal{f} 1993 ; 6$ (Suppl 16): $1-100$.

8 Black LF, Hyatt RE. Maximal respiratory pressures: normal values and relationship to age and sex. Am Rev Respir Dis 1969;99:696-702.

9 Braun NMT, Arora NS, Rochester DF. Respiratory muscle and pulmonary function in polymyositis and other proximal myopathies. Thorax 1983;38:616-23.

10 Rochester DF. Tests of respiratory muscle function. Clin Chest Med 1988;9:249-61. 
11 Whitelaw WA, Derenne JP, Milac-Emili J. Occlusion pressure as a measure of respiratory center output in conscious man. Respir Physiol 1975;23:181-99.

12 Gorini M, Ginanni R, Spinelli A, Duranti R, Andreotti L Scano G. Respiratory muscle strength and respiratory drive in patients with rheumatoid arthritis. Am Rev Respir Dis 1990;142:289-94.

13 Gorini M, Spinelli A, Ginanni R, Duranti R, Gigliotti F Scano G. Neural respiratory drive and neuromuscular coupling during $\mathrm{CO}_{2}$ rebreathing in patients with chronic interstitial lung disease. Chest 1989;96:824-30.

14 Spinelli A, Marconi G, Gorini M, Pizzi A, Scano G. Contro of breathing in patients with myasthenia gravis. $\mathrm{Am} \mathrm{Rev}$ Respir Dis 1992;145:1359-66.

15 Gross D, Grassino A, Ross WRD, Macklem PT. Electromyogram pattern of diaphragmatic fatigue. $\mathcal{F} A p p l$ Physiol 1979;46:1-7.

16 Lopata M, Evanich MJ, Lourenco MV. Quantification of diaphragmatic EMG response to $\mathrm{CO}_{2}$ rebreathing in diaphragmatic EMG response to $\mathrm{CO}_{2}$
humans. $₹$ Appl Physiol 1977;43:262-70.

17 Read DJC. A clinical method for assessing ventilatory response to carbon dioxide. Australas Ann Med 1967;16: 20-32.

18 Demedts M, Beckers J, Rochette F, Bulcke JA. Pulmonary function in moderate neuromuscular disease without respiratory complaints. Eur Respir f 1982;63:62-7.

19 Pride NB. Interactions between chest wall, respiratory muscles and lung function in disease. Bull Eur Physiopathol Respir 1984;20:423-8.

20 De Troyer A, Borenstein S, Cordier R. Analysis of lung volume restriction in patients with respiratory muscle weakness. Thorax 1980;35:603-10.

21 Estenne M, Gevenois PA, Kinnear W, Soudon P, Heilporn $\mathrm{A}, \mathrm{De}$ Troyer A. Lung volume restriction in patients with chronic respiratory muscle weakness: the role of microatelectasis. Thorax 1993;48:698-701.

22 Stubgen JP, Ras GJ, Schultz GM, Crowther G. Lung and respiratory muscle function in limb-girdle muscular dystrophy. Thorax 1994;49:61-5.

23 Begin R, Bureau MA, Lupien L, Lemieux B. Control of breathing in Duchenne's muscular dystrophy. $A m ₹ \mathrm{Med}$ 1980;69:227-34.

24 Begin R, Bureau MA, Lupien L, Bernier JP, Emieux B. Pathogenesis of respiratory insufficiency in myotonic dystrophy. Am Rev Respir Dis 1982;125:312-8.

25 Serisier DE, Mastaglia FI, Gibson GJ. Respiratory muscle function and respiratory control. I. In patients with motorneuron disease. II. In patients with myotonic dystrophy. Of Med 1982;202:205-26.

26 Gilmartin J, Walls TJ, Stone TN, Gibson GB. Relationship between ventilatory and mouth occlusion pressure responses to carbon dioxide in normal subjects and patients with muscle weakness. Thorax 1980;35:716.

27 Gibson GJ, Pride NB, Newsom-Davis J, Loh LC. Pulmonary mechanics in patients with respiratory muscle weakness. Am Rev Respir Dis 1977;115:389-95.

28 Rochester DF. Respiratory muscle weakness, pattern of breathing, and $\mathrm{CO}_{2}$ retention in chronic obstructive pulbreathing, and $\mathrm{CO}_{2}$ retention in chronic obstructive

29 Evanich MJ, Bruce E, Eldridge FL, Goldman M, Lopata $\mathrm{M}$, Lourenco RV, et al. Conference report: Workshop on assessment of respiratory control in humans: IV. Measurement of the electrical activity in respiratory muscles. $A m$ Rev Respir Dis 1977;115:541-8.

30 Lopata M, Pearle J. Diaphragmatic EMG and occlusion pressure response to elastic loading during $\mathrm{CO}_{2}$ rebreathing in humans. 7 Appl Physiol 1980;49:669-75.

31 Smith PEM, Calverley PMA, Edwards RHT, Evans GA Campbell EJM. Practical problems in the respiratory care of patients with neuromuscular dystrophy. $N$ Engl $₹$ Med of patients with neur.

32 Holle RHO, Schoene RB, Pavlin EJ. Effect of respiratory muscle weakness on $\mathrm{P} 0 \cdot 1$ induced by partial curarization. f Appl Physiol 1984;57:1150-7.

33 Grinman S, Whitelaw WA. Pattern of breathing in case of generalized respiratory muscle weakness. Chest 1983;84: 770-2.

34 Shannon $R$. Reflexes from respiratory muscles and costovertebral joints. In: Cherniack NS, Widdicombe JG, eds. Handbook of physiology: The respiratory system. Vol II. Control Handbook of physiology: The respiratory system. Vol II. Control
of breathing. Part 1. Bethesda: American Physiological of breathing. Part 1.

35 Riley DJ, Santiago TV, Daniele RP, Edelman NH. Blunted respiratory drive in congenital myopathy. $A m \mathcal{F}$ Med 1977 63:459-66.

36 Gillam PMS, Heaf PJD, Kaufman L, Lucas BGB. Respiration in dystrophia myotonica. Thorax 1964;19:112 120.

37 Renzi J, Milic-Emili J, Grassino AE. The pattern of breathing in diffuse lung fibrosis. Bull Eur Physiopathol Respir 1982; 18:461-72.

38 Vinken W, Elleker G, Cosio MG. Detection of upper airway muscle involvement in neuromuscular disorders using the flow-volume loop. Chest 1986;90:52-7.

39 Epstein SK. An overview of respiratory muscle function. In: Fanburg BL, Sicilian LS, eds. Respiratory dysfunction in neuromuscular disease. Clin Chest Med 1994;15 619-39.

40 Whitelaw WA, Derenne JP. Airway occlusion pressure. $f$ Appl Physiol 1993;74:1475-83. 